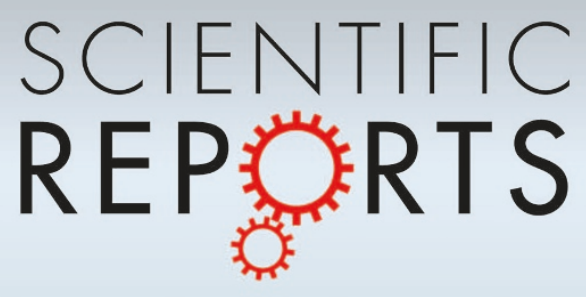

OPEN

SUBJECT AREAS:

OPTICAL IMAGING

OPTOGENETICS

BIOLOGICAL FLUORESCENCE

TECHNICAL REPORT

Received

13 May 2013

Accepted

21 June 2013

Published

19 July 2013

Correspondence and requests for materials should be addressed to T.K. (tknopfel@knopfellab.net)

* The authors contributed equally to this work.

\section{Two-photon voltage imaging using a genetically encoded voltage indicator}

\author{
Walther Akemann ${ }^{1 *}$, Mari Sasaki ${ }^{2 *}$, Hiroki Mutoh', Takeshi Imamura ${ }^{2}$, Naoki Honkura $^{2}$ \\ \& Thomas Knöpfel ${ }^{1,3}$
}

${ }^{1}$ RIKEN Brain Science Institute, Hirosawa, Wako-City, 35 1-0198 Saitama, JAPAN, ${ }^{2}$ Ehime University Graduate School of Medicine, Shitsukawa, Toon, 791-0295 Ehime, JAPAN, ${ }^{3}$ Imperial College Faculty of Medicine, London W12 oNN, UK.

Voltage-sensitive fluorescent proteins (VSFPs) are a family of genetically-encoded voltage indicators (GEVIs) reporting membrane voltage fluctuation from genetically-targeted cells in cell cultures to whole brains in awake mice as demonstrated earlier using 1-photon (1P) fluorescence excitation imaging. However, in-vivo 1P imaging captures optical signals only from superficial layers and does not optically resolve single neurons. Two-photon excitation (2P) imaging, on the other hand, has not yet been convincingly applied to GEVI experiments. Here we show that $2 \mathrm{P}$ imaging of VSFP Butterfly 1.2 expresssing pyramidal neurons in layer $2 / 3$ reports optical membrane voltage in brain slices consistent with $1 \mathrm{P}$ imaging but with a 2-3 larger $\Delta \mathrm{R} / \mathrm{R}$ value. $2 \mathrm{P}$ imaging of mouse cortex in-vivo achieved cellular resolution throughout layer $2 / 3$. In somatosensory cortex we recorded sensory responses to single whisker deflections in anesthetized mice at full frame video rate. Our results demonstrate the feasibility of GEVI-based functional $2 \mathrm{P}$ imaging in mouse cortex.

rains use temporal structure of action potential sequences in large populations of neurons to code complex information for the control of behavior ${ }^{1-3}$. Data from many brain systems meanwhile show that temporal correlations in action potential output of neurons are often associated with robust synchrony of membrane voltage fluctuation reflecting common time structure of received synaptic input as well as dynamic properties of the imbedding circuits ${ }^{4-6}$. The spatial-temporal structure of membrane voltage fluctuation in large-scale cortical networks is principally assessable to voltage imaging using potentiometric indicators designed to emit a fluorescence signal sensitive to the local trans-membrane voltage when excited by light ${ }^{7,8}$. The most recent class of voltage indicators consists of DNA-encoded protein probes (genetically encoded voltage indicators; GEVI) ${ }^{9-14}$ offering several improvements over low molecular weight voltage sensitive dye indicators, such as absence of phototoxic and pharmacological effects, stable long-term expression and the ability to target specific cell types ${ }^{15}$. GEVI imaging supports the functional dissection of neuronal circuits using techniques that are fully compatible with optogenetic tools ${ }^{16,17}$. Most of recent GEVI variants use the voltage sensor domain of voltage-activated phosphatase ${ }^{12,13,18,19}$, mostly Ci-VSP (Ciona voltage-sensitive phosphatase), as a voltage-sensitive component together with fluorescent protein at insertion sites suitable to render the fluorescence emission sensitive to conformation changes performed by the voltage-sensor domain. The very first GEVIs using the Ci-VSP voltage sensor was the VSFP2 series of probes (VSFP; voltage-sensitive fluorescent protein) which achieved reliable function in neuronal cell culture, brain slice and mouse brain in-vivo, ${ }^{9,21}$. Recently, we introduced VSFP Butterfly 1.2, an enhanced VSFP variant featuring a mCitrine/mKate2 FRET reporter with the mCitrine donor linked to S4 and the mKate2 acceptor to the S1 transmembrane segment of the Ci-VSP voltage sensor. VSFP Butterfly became the first GEVI to provide sufficient sensitivity to report not only sensory population responses in somato-sensory cortex with high signal-to-noise in-vivo, but also spontaneous cortical network activity across different brain states ${ }^{12}$.

Voltage imaging using low molecular weight fluorescent voltage-sensitive dye indicators in wide field mode was extensively applied to cortical information processing, e.g. to reveal sensory feature representations in different operating states of cortical networks ${ }^{22-25,25-29}$. However, wide field images of cortex stained with fluorescent indicators lack cellular resolution because of the blur produced by out-of-focus light and light scattering. The optically reported membrane voltage, therefore, reflects an average over all stained membranes within a tissue volume containing a large number of cells. While monitoring compound activities can provide a useful representation of cortical dynamics by emphasizing the major modes of activity shared by a larger set of neurons, other questions require cellular resolution. The foremost optical approach to improve spatial resolution and 
deliver un-blurred optical sections of micrometer thickness up to larger tissue depth is 2-photon microscopy ${ }^{30}$. 2-photon voltage imaging, however, has been lagging as imaging method, mostly because of the low photon counts expected from sequential image scan acquisition at fast frame rate, unavoidably leading to high photon shot noise ${ }^{31}$. Notwithstanding, in-vitro studies recently demonstrated single-trial sensitivity for recording of action potentials in axonal terminal arbors ${ }^{32}$, back-propagated action potentials in single spines ${ }^{33}$ and spontaneous and evoked somatic potentials in neurons in acute brain slice ${ }^{34}$ using 2-photon imaging of voltagesensitive dyes. On the other hand, available 2-photon imaging data of sensory-evoked activity in somatosensory and visual cortex remained poor in signal-to-noise requiring extensive trial averages $(>100)$ to surpass the noise levels ${ }^{31,35}$.

We speculated that the latest generation of VSFP indicators would be suitable for in-vivo 2-photon voltage imaging of cortical electrical activity at favorable SNR by taking advantage of several factors, in particular high gain of the indicator and exclusive expression in targeted cells. By expressing VSFP Butterfly 1.2 in layer 2/3 pyramidal neurons and using standard microscope hardware, we obtain two- to threefold larger fractional indicator responses $\left(\Delta \mathrm{F} / \mathrm{F}_{0}\right.$ and $\Delta \mathrm{R} / \mathrm{R}_{0}$ values) in 2-photon versus 1-photon excitation imaging and a sufficient SNR to record cellular responses in-vitro and bulk population responses in-vivo in single trials.

\section{Results}

In previous work, we developed a set of in-vitro assays to evaluate VSFP probes for their general photophysical properties and physiological utility under 1-photon excitation. As for these, the VSFP Butterfly 1.2 is perhaps the best characterized GEVI probe available today, with a documented maximal voltage sensitivity of $22 \%$ per $100 \mathrm{mV}$ close to half activation voltage of $-70 \mathrm{mV}$ and a response time with a leading component of 2-6 $\mathrm{ms}^{12}$. The utility of VSFPs to probe physiological processes in 2-photon imaging, however, has not been addresses before. Most importantly, this concerns the magnitude of the 2-photon excited optical voltage report in brain tissue and the overall signal-to-noise ratio (SNR) achievable in physiological experiments. To answer these questions, we first performed imaging experiments on cortical brain slices from adult mice expressing Butterfly 1.2 in a dense population of pyramidal neurons in cortical layer $2 / 3$ following in-utero electroporation of pCAGButterfly 1.2 plasmid at E14.5 to E15.5 (see ${ }^{20}$ and Methods). To compare the probe response in the same sample under 1-photon and 2-photon excitation we set up the microscope to support both imaging modes. By applying a brief burst stimulus ( 5 pulses; $100 \mathrm{~Hz}$ ) to a microelectrode placed in layer 4 , we first imaged the mCitrine fluorescence from a large field in layer2/3 using 1-photon excitation at $495 \mathrm{~nm}$ (Fig. 1a) yielding an optical signal of $0.3 \%$ fractional mCitrine fluorescence response $\left(\Delta \mathrm{F} / \mathrm{F}_{0}\right)$ in the example shown (Fig. $1 \mathrm{~b}$ and c). Regarding efficient 2-photon excitation, we note that mCitrine has a peak 2-photon absorption at $950 \mathrm{~nm}^{36}$. Limited by the working range of our Ti-Sapphire laser, we set the excitation wavelength slightly below $950 \mathrm{~nm}$ centering around $920 \mathrm{~nm}$ in this set of experiments. To scan the sample at video rate, we scanned a smaller field through the same $20 \times$ objective (red frame in Fig. 1a) at $32 \times$ 32 pixel resolution while applying the 5 pulse stimulus as before. Expectedly, the 2-photon image resolved the dense packing of fluorescent neurites in layer $2 / 3$ (Fig. 1d), while the fluorescence signal exhibited a decrease of mCitrine and an increase of mKate 2 emission (Fig. 1e) as with 1-photon excitation ${ }^{12}$. Although the 2-photon recordings carried substantially more baseline-normalized noise, the amplitude of the fractional fluorescence response was about $1 \%$ in the mCitrine channel, notably larger than in 1-photon mode $(\sim 0.3 \%)$, and increased to about $3 \%$ in the presence of the GABA(A) receptor antagonist Gabazine. Under Gabazine the response was easily revealed with good SNR $(\sim 2-3)$ in single trials (Fig. 1f), facilitated by a higher level of stimulus-induced activity in the partially disinhibited network. The example shown in Fig. 1 was a

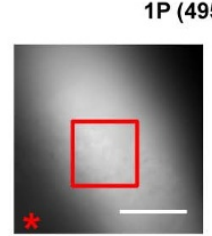

b

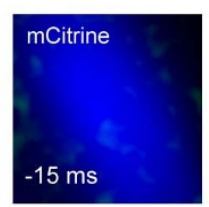

C
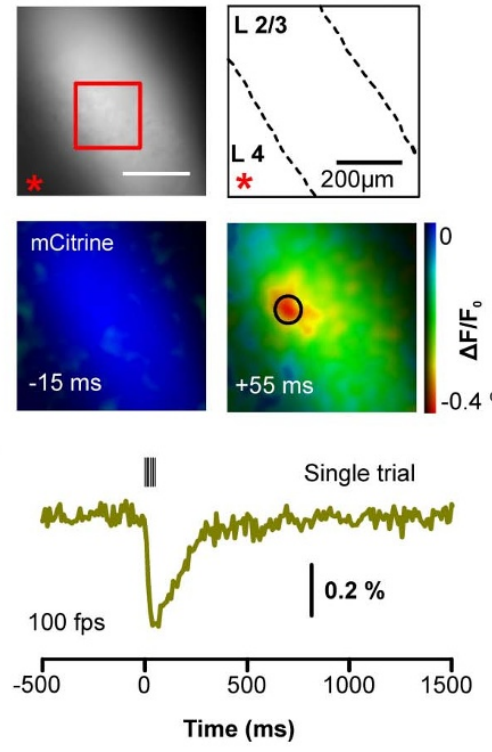

d
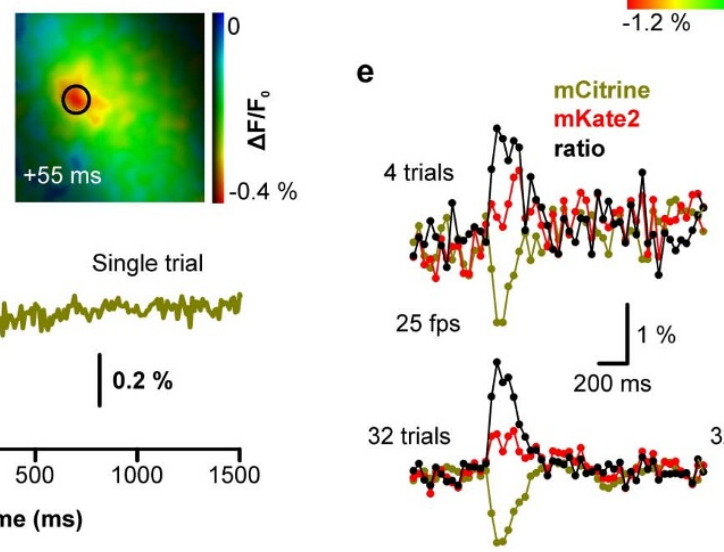

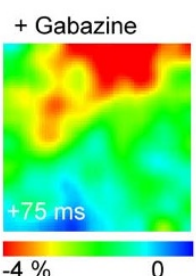

f + Gabazine $(10 \mu \mathrm{M})$

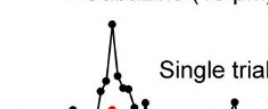

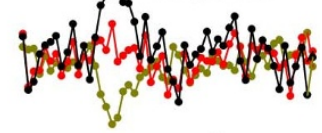

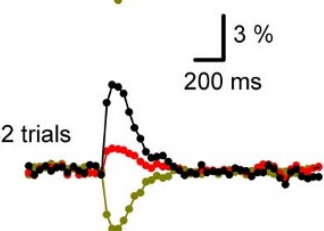

Figure $1 \mid$ Evaluation of Butterfly 1.2-reported neuronal electrical population activity recorded in $1 \mathrm{P}$ and $2 \mathrm{P}$ excitation mode. (a) $1 \mathrm{P}$-exited mCitrine image of a coronal brain section showing cortical layer $2 / 3$ as a bright band. (b) Color-coded fractional fluorescence response $\left(\Delta \mathrm{F} / \mathrm{F}_{0}\right)$ in response to a 5 pulse $(100 \mathrm{~Hz})$ stimulus applied through a stimulation electrode placed in layer 4 (red asterisk). (c) Time course of Butterfly 1.2 response taken from the region of interest marked by black circle in (b). (d) Left: 2P-excited mCitrine image of an area lined out by red frame in (a). Middle: Fractional mCitrine fluorescence in response to the same stimulus as used before. Right: Response after bath application of the $\mathrm{GABA}_{\mathrm{A}}$-receptor antagonist Gabazine $(10 \mu \mathrm{M})$. (e) Time course of $2 \mathrm{P}$-excited mCitrine (yellow), mKate2 (red) and mKate2/mCitrine ratio (black) signals (4 trial and 32 trial averages) corresponding to the region of interest indicated in (d) in response to a 5 pulse stimulus. (f) Same in (e) after Gabazine (10 $\mu \mathrm{M}$ ) application. 
consistent with all preparations tested, although SNR varied with the level of Butterfly expression. To further improve SNR, we changed from linear to spiral scan, which permitted two- to threefold longer dwell time at similar frame rate and pixelation (Fig. 2). In the example of the well-resolved superficial cell in Fig. 2a the largest absolute mCitrine response $(\Delta \mathrm{F})$ appeared at the location of the cell body and in a proximal neurite field where the evaluated amplitude of mKate $2 / m$ Citrine ratio response $\left(\Delta \mathrm{R} / \mathrm{R}_{0}\right)$ reached up to $3 \%$ (Fig. 2b). Here, the ratio response is presented as color-coded overlay onto the mCitrine fluorescence gray scale image for all pixels where the absolute mCitrine response, after spatial filtering, reached a $\mathrm{z}$ score above 2 above baseline noise. As the absolute response is expected to be large in responsive pixels of high brightness, thresholding by the absolute fluorescence response allowed us to trace single responsive cells. When extracting the mKate $2 / \mathrm{mCitrine}$ signal from a cell body (yellow circle in Fig. 2a and b), voltage signals were robustly seen in single trials (Fig. $2 \mathrm{c}$ and $\mathrm{d}$ ).

The above data suggest that 2-photon VSFP imaging of neuronal activity is feasible up to single-trial sensitivity in brain slices where the only limiting source of noise is the photon quantum statistics itself. Next, we set out to determine whether the probe would afford detection of sensory-evoked electrical activity in-vivo where additional noise may arise from tissue movement and hemodynamic effects. We performed in-vivo imaging of whisker deflection-induced voltage responses in barrel cortex of anesthetized and head-fixed adult mice. Upon directing a brief air puff focally onto the $\mathrm{C} 1$ whisker we first identified the location of the cortical responsive area by macroscopic trans-cranial voltage imaging of the contralateral cortical hemisphere using 1-photon excitation as described ${ }^{20}$. Once the $\mathrm{C} 1$ responsive area was mapped (Fig. $3 \mathrm{e}$ and $\mathrm{f}$ ), we performed a small circular craniotomy centering around the $\mathrm{C} 1$ barrel column for optimal optical access. We then imaged the underlying cortex consecutively in 1-photon (Fig. 3g) and 2-photon (Fig. 3h) excitation mode, with identical fields of view (Fig. $3 \mathrm{c}$ and d), using an optical configuration as schematically indicated in Fig. 3a. In response to the whisker stimulation, responsive areas and time courses (Fig. $3 \mathrm{~g}$ and h) were consistent between both imaging modalities (Fig. $3 \mathrm{~g}$ ). The optical signal sampled over an area where the absolute mCitrine response $(\Delta \mathrm{F})$ supersedes a threshold of $\mathrm{z}$-score 1.5 (corresponding to a region of interest defined by the colored area at the time of peak
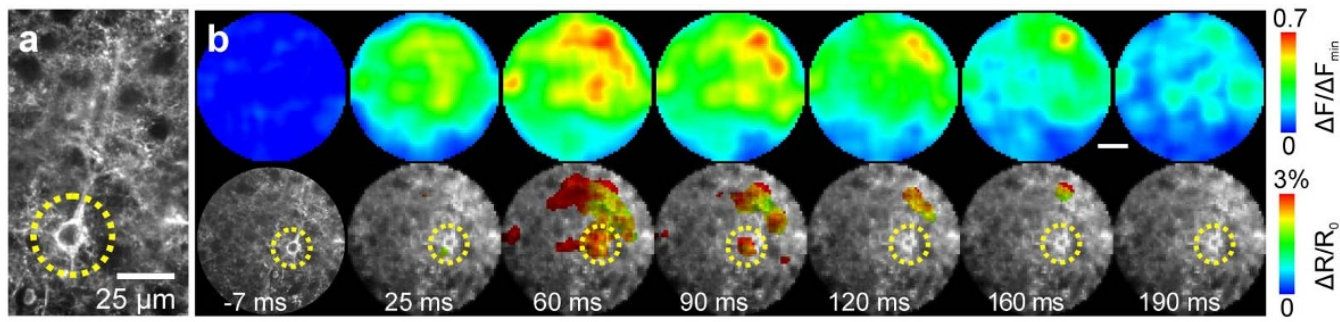

C

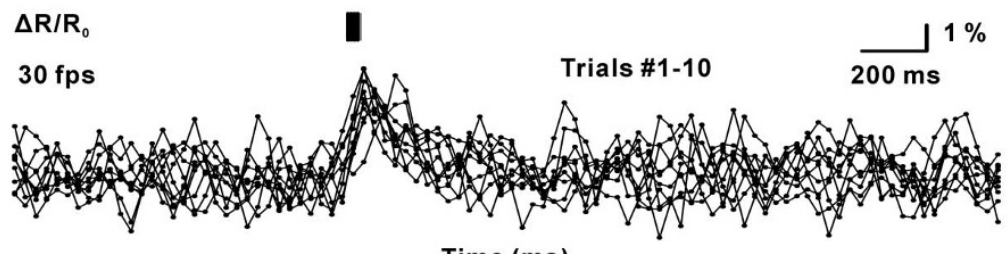

d

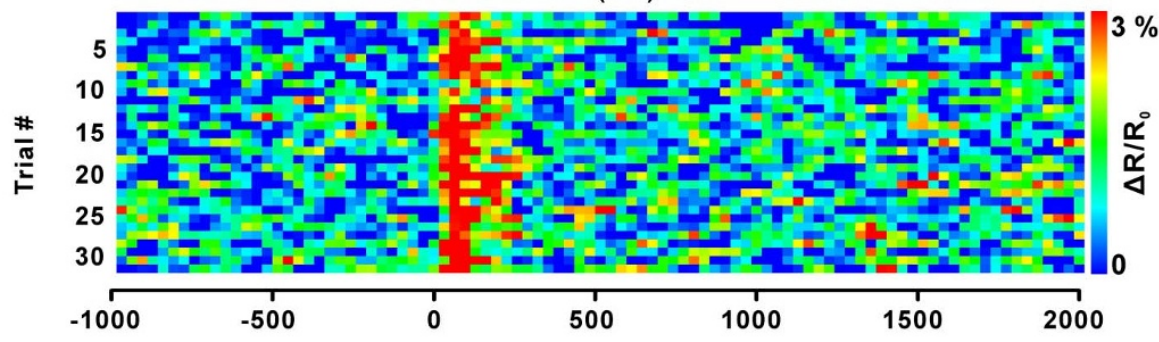

e

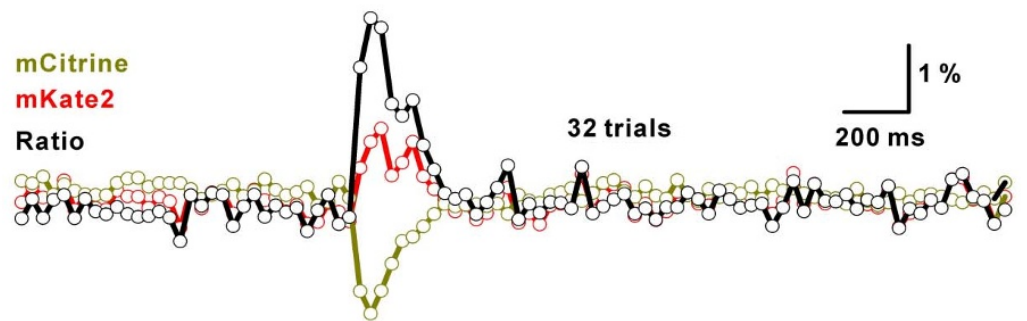

Figure 2 $2 \mathrm{P}$ imaging of single cell voltage response in-vitro. (a) $2 \mathrm{P}$ mCitrine fluorescence image of a layer $2 / 3$ pyramidal cell in a cortical brain slice $120 \mu \mathrm{m}$ below slice surface. (b) Time lapse series of Butterfly 1.2 fluorescence images in response to a 5 pulse (100 Hz) extracellular current stimulus applied at time zero. Upper row: Absolute differential mCitrine response $\left(\Delta \mathrm{F} / \Delta \mathrm{F}_{\text {min }}\right)$ at different times after stimulus presentation.

Lower row: mCitrine fluorescence image (grey) overlaid with the color-coded mKate $2 / \mathrm{mCitrine}$ response $\left(\Delta \mathrm{R} / \mathrm{R}_{0}\right)$ of those pixels yielding a mCitrine response amplitude $(\Delta \mathrm{F})$ of $\mathrm{z}$-score of two over mean baseline fluctuation $\left(\Delta \mathrm{F}_{0}\right)$ across the whole image area. (c) Time course of the trial number 1 to 10 taken from a region of interest surrounding a pyramidal cell body (indicated yellow in a and b). (d) Trail overview showing the color-coded mKate2/ mCitrine ratio signal $\left(\Delta \mathrm{R} / \mathrm{R}_{0}\right)$ of the cell body (yellow circle in a and b) of all 32 trials. (e) Time course of the mCitrine (yellow), mKate2 (red) and mKate2/ mCitrine ratio in the trial average. 
a

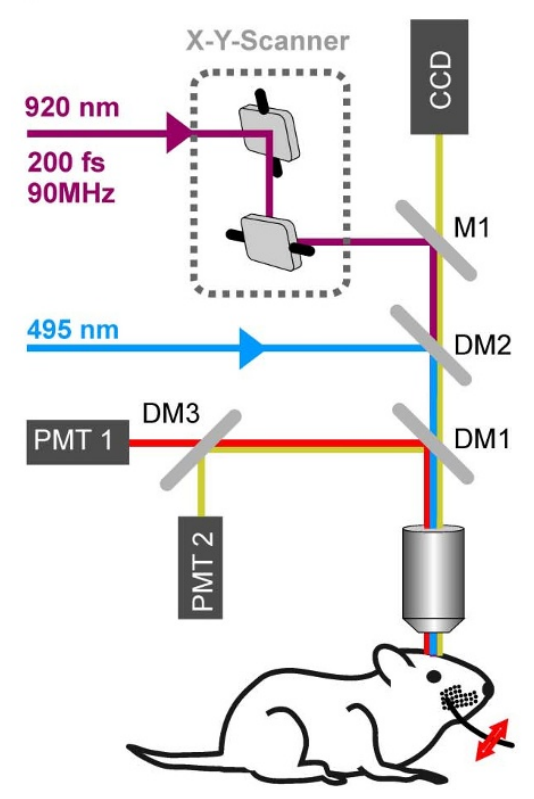

b

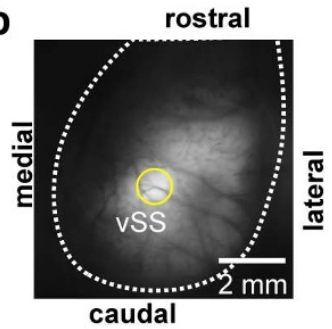

e

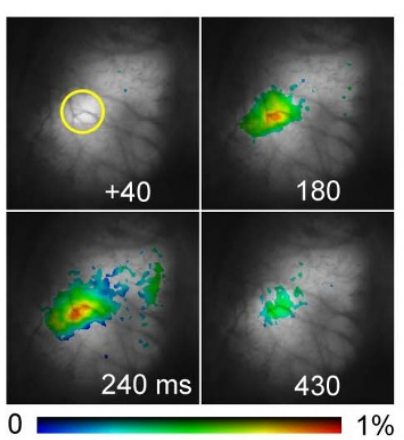

C

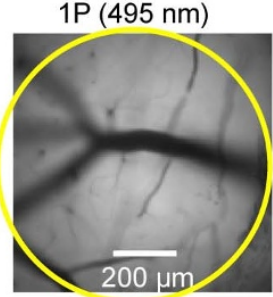

f d

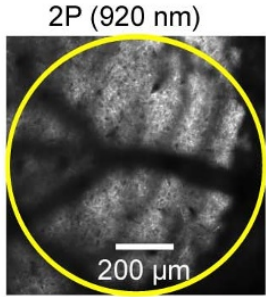

$\Delta \mathrm{R} / \mathrm{R}_{0}$

g

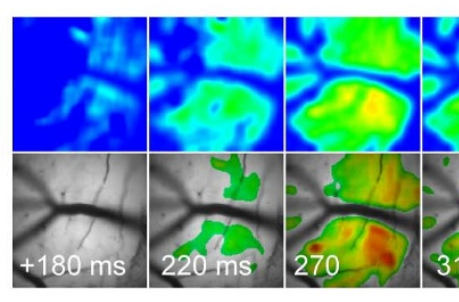

1P $(495 \mathrm{~nm})$

i

$2 \mathrm{P}(920 \mathrm{~nm})$

$1 \mathrm{P}(495 \mathrm{~nm})$

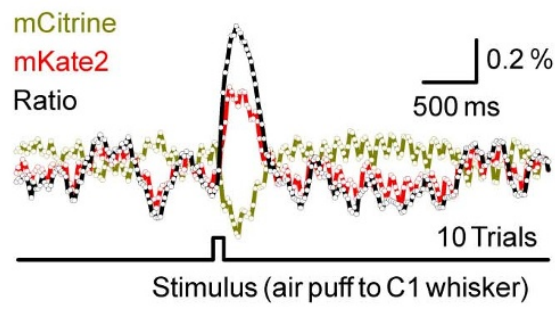

h

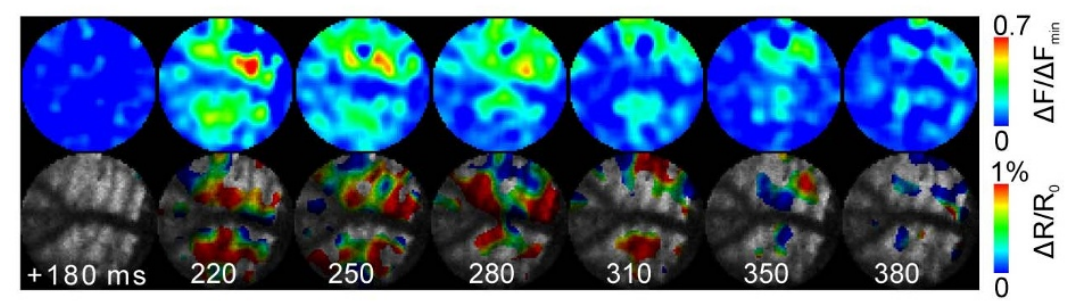

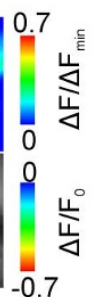

$-0.7$
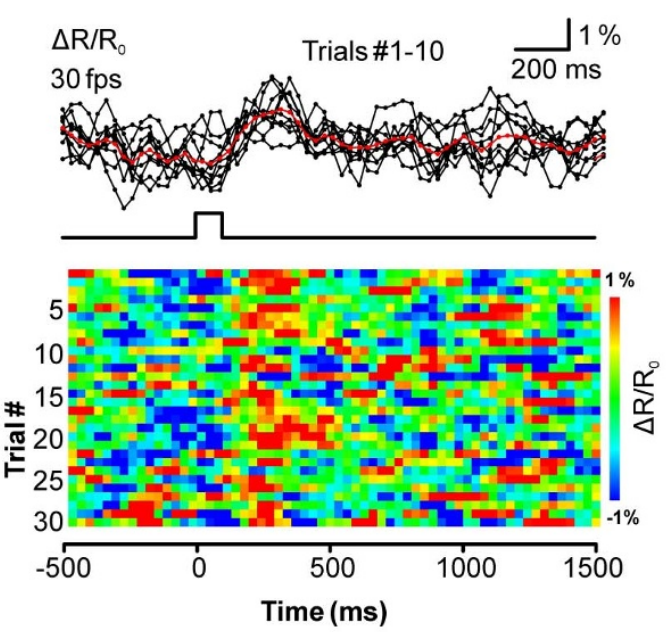

Figure $3 \mid 2 \mathrm{P}$ imaging of the population voltage responses in the barrel cortex in-vivo. (a) Experimental setup permitting $1 \mathrm{P}$ and $2 \mathrm{P}$ voltage imaging of head-fixed mice. (b) Macroscopic mCitrine 1P fluorescence image showing the left cortical hemisphere of an 8 weeks old mouse. (c) mCitrine $1 \mathrm{P}$ image of the $\mathrm{C} 1$ barrel cortex after cranitomy. The yellow circle marks the area covered by the spiral laser scan in $2 \mathrm{P}$ imaging mode. (d) mCitrine $2 \mathrm{P}$ image of the field as in (c) $190 \mu \mathrm{m}$ below pia. (e) Visualization of the $\mathrm{C} 1$ barrel response after craniotomy in $1 \mathrm{P}$ whole hemisphere imaging showing the mCitrine baseline fluorescence (gray) overlaid with the color-coded mKate2/mCitrine response at different times relative to stimulation time. (f) Time course of the mCitrine (yellow), mKate2 (red) and mKate $2 / m$ Citrine ratio signals recorded from the region of interest (yellow circle) indicated in (e). (g) Time lapse series of the Butterfly 1.2-reported cortical response to $\mathrm{C} 1$ whisker stimulation imaged in the 1P mode. Upper row: Absolute differential mCitrine response $\left(\Delta \mathrm{F} / \Delta \mathrm{F}_{\mathrm{min}}\right)$. Lower row: $\mathrm{mCitrine}$ fluorescence image (grey scale) overlaid with the color-coded mKate $2 / \mathrm{mCitrine}$ response of pixels yielding a mCitrine response amplitude $(\Delta \mathrm{F})$ of $\mathrm{z}$-score of 1.8 over mean baseline fluctuation $\left(\Delta \mathrm{F}_{0}\right)$ across the whole image area after averaging 32 trials. (h) $2 \mathrm{P}$ excitation recording with the same field of view as in (g). (i) Time course of $2 \mathrm{P}$ mKate $2 / \mathrm{mCitrine}$ signals recorded from the region of interest comprising all color-coded pixels of the peak response shown in h (lower row). Top: Overlay of first 10 trials together with the mean (red). Bottom: Overview of all 32 trials showing the $\Delta \mathrm{R} / \mathrm{R}_{0}$ response according to the indicated color code.

response) was revealed in single trials (Fig. 3i). It is important to note that the fluctuation of the baseline signal in this case also includes the voltage signals reporting ongoing spontaneous electrical activity ${ }^{12}$ in addition to photon shot noise.

The power of functional optical imaging derives from the ease to adapt the magnification to different spatial scales of interest. The 2photon imaging experiments described above aimed at population activity using low magnification and large-scale spatial filtering. In a second set of experiments, we explored the Butterfly response at cellular resolution using a $25 \times$ objective and full frame $(512 \times$ 512) resonance scanning at video rate. In this setting, single cell somata (Fig. 4a) and dendritic branches (Fig. 4b) were easily resolved in-vivo in all 10 animals imaged. In 5 out of 10 animals we identified individual cells that were responsive to a whisker deflection stimulus (marked with asterisks in Fig. $4 \mathrm{~b}$ and $\mathrm{d}$ ) when accumulating the signal of the brightest pixels over a few trials. In two recordings, 
we also probed the voltage response to a stimulus applied to a firstorder neighboring whisker and did not detect a signal above noise level (Fig. $4 \mathrm{~b}$ and d) indicating sharp receptive fields. This is in agreement with intracellular recordings where a twofold decrease of EPSP amplitude in barrel cortex neurons was found in response to first-order neighbor versus principal whisker stimulation ${ }^{37,38}$. The existence of a principal whisker and absence of neighboring whisker signals offers an internal validation of these measurements that let us rule out relevant contributions of stimulus artifacts.

\section{Discussion}

The present work demonstrates the feasibility of GEVI-based 2photon voltage imaging as a tool to map electrical activity in intact brain circuits. We obtained sufficient SNR to permit imaging of sensory-evoked population activity in high-resolved optical sections of layer $2 / 3$ in-vivo.

The optical voltage signals in these 2 -photon recordings have a dynamic range of 1 to $3 \%$, about 2 to 3 times larger than corresponding 1-photon recording, but 1 to 2 orders of magnitude smaller than typical calcium imaging signals. However, calcium imaging provides a very indirect measure of neuronal activity, generally limited to supra-threshold activity and certain cell types ${ }^{39-41}$. While action potentials can produce large calcium transients in axon terminals, cell body and parts of dendrites, slow subthreshold potentials produce orders of magnitude smaller calcium signals ${ }^{39,42}$. Voltage indicators including GEVIs principally afford broader coverage of neuronal activity, including sub threshold potentials. VSFP Butterfly 1.2 , specifically, responds with highest gain (slope of fluorescenceto-voltage relationship) to voltage fluctuations around the resting membrane voltage ${ }^{12}$. Although Ci-VSP-derived voltage indicators can report fast action potentials ${ }^{12,18,20}$, the optical signal is generally small because fast somatic action potentials activate the sensor only partially ${ }^{43}$. We, therefore, interpret the Butterfly 1.2 signal as reporting mostly somato-dendritic activity due to post synaptic potentials and likely smaller contributions from backpropagating action potentials and fast dendritic spikes. For these reasons, voltage and calcium imaging capture largely complementary aspects of the neuronal dynamics ${ }^{42}$.

In 2-photon scanning microscopy (TPSM), photons are collected from a small $(<1 \mu \mathrm{m})$ excitation volume in the laser focus where the electromagnetic field strength is large enough to yield significant 2photon absorption of the probe $e^{30,44}$. The thickness of the focal plane in TPSM equals the axial dimension of the effective 2-photon point spread function in the order of microns depending on wavelength and numerical aperture of the objective. Since photo-absorption outside of this plane is very low, TPSM delivers practically background-free images of micron-sized optical sections of thick tissue ${ }^{44}$. In our experiments, we could obtain cellular resolution images up to the border of layer $2 / 3$ to layer 4 spanning the whole cortical sheet of Butterfly 1.2-expressing cells. Suppression of out-of-focus fluorescence background explains why the functional response had 2-3 times larger apparent amplitude when acquired in 2-photon versus 1-photon excitation mode, even in the case of large regions of interest comprising many cells (Fig. 1 and 3). As the SNR of the optical measurement is a linear function of the apparent response magnitude ${ }^{43}$, these gains help overcome the detection threshold set by the higher level of photon quantum noise in scanning 2-photon microscopy.

This work presents the first optical voltage measurement by TPSM using a Ci-VSP based GEVI reporter in cortical cells. Another study recently reported TPSM of Flare-reported cellular resolution signals in visual cortex ${ }^{35}$. However, the signals responding to visual stimuli were very weak, requiring extensive trial averaging $(>100)$, while lacking convincing validation to proof that these signals reliably represent membrane voltage, given that Flare and other first generation GEVIs, derived from voltage-gated ion channels, were not
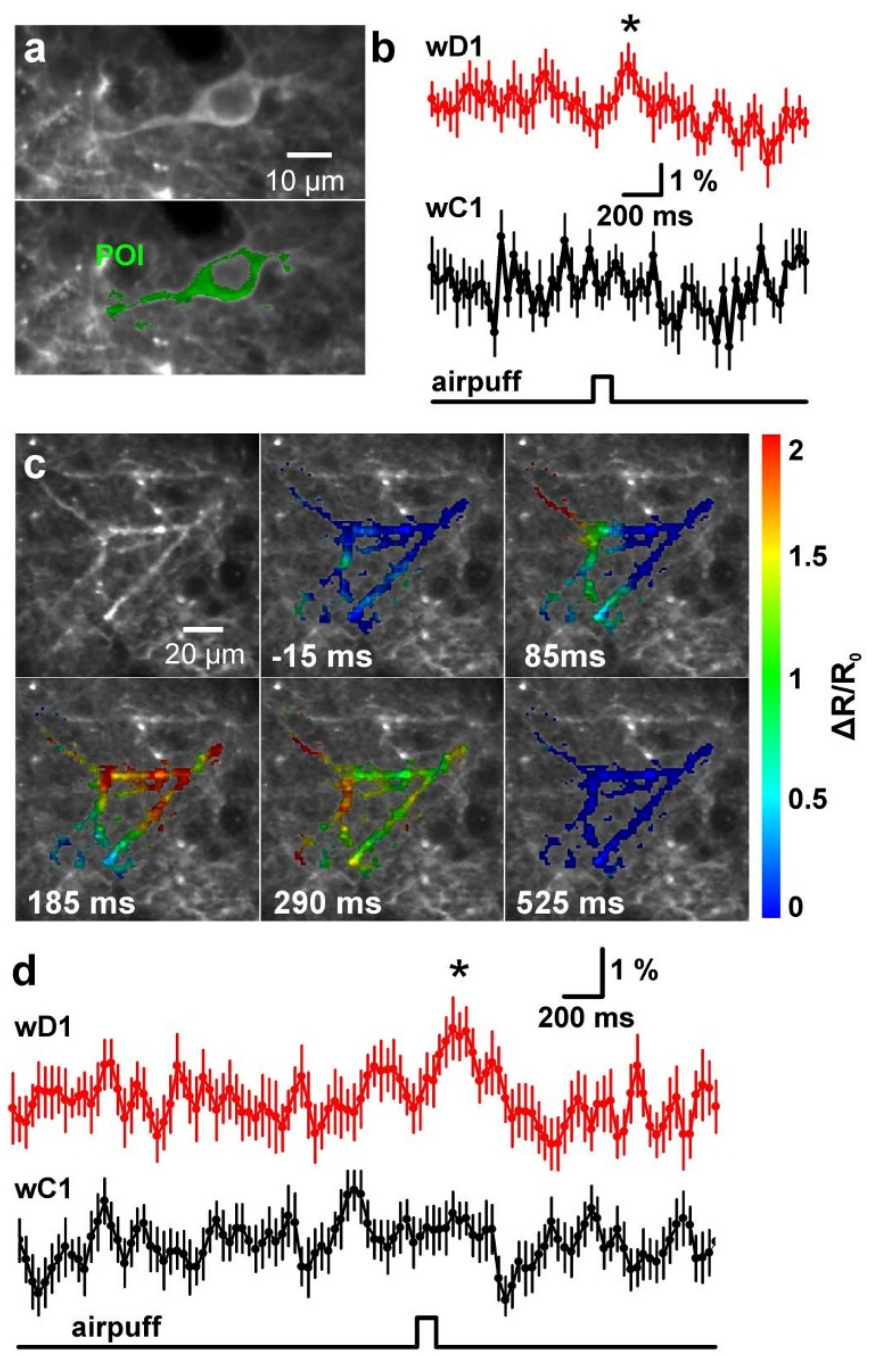

Figure $4 \mid$ Tactile responses in the barrel cortex at cellular resolution. (a) $2 \mathrm{P}$ image of a layer2/3 pyramidal cell expressing Butterfly 1.2 in-vivio (top) with pixels of interest (POI) marked in green (buttom). (b) Time course of the $2 \mathrm{P}$ mKate/mCitrine ratio $\left(\Delta \mathrm{R} / \mathrm{R}_{0}\right)$ signal in response to $\mathrm{D} 1$ whisker (wD1; red) and $\mathrm{C} 1$ whisker (wC1; black) stimulus after averaging 16 trials, with error bars representing SEM and the time of the stimulus trigger signal indicated at the bottom. D1 response marked by asterisk. (c) Another field of view from the same animal preparation showing segments of dendrites with the color-coded ratio response $\left(\Delta R / R_{0}\right)$ superimposed onto the mCitrine image at pixels of highest brighness selected with a $\mathrm{z}$ score of 1.3 from the mCitrine image, with the time measured from the onset of the stimulus trigger signal. (d) Time course of the response to D1 whisker (red) and $\mathrm{C} 1$ whisker stimulation (black) with pixels colored in (c) taken as the region of interest and the time of the stimulus trigger signal indicated at the bottom. Average over 16 trials with error bars representing SEM.

functional when tested in cultured mammalian cells ${ }^{45}$. CiVSP-based, second generation indicators such as VSFP2s and VSFP-Butterflies, on the other hand, were extensively validated and show consistent function in all preparations studied so far, including cultured neurons, brain slices and whole brain in-vivo ${ }^{13,20}$, in 1 -photon and 2photon excitation.

The present data provide the proof-of-principle for 2-photon VSFP voltage imaging using standard TPSM. To achieve sufficient SNR we used spatial filtering to accumulate the photon counts across neighboring pixels, thereby scarifying spatial resolution. A promising alternative hardware-based approach to increase the photon sampling rate uses temporal focusing of the excitation light to permit 
a larger excitation area without loss of axial resolution ${ }^{46}$. Another approach implements 3D random-access scanning to increase dwell times and hence photon yields at individual voxels of interest ${ }^{47,48}$. We expect that a combination of innovative optical methods, requiring specialized optical hardware, will further improve the SNR in VSFP 2-photon voltage imaging and facilitate long-term, non-invasive monitoring of membrane voltage signaling in cortical circuitry at high spatial resolution in-vivo.

\section{Methods}

Animal welfare. Animal experiments were performed in accordance with the National Institutes of Health guidelines for animal research and were approved by the Institutional Animal Care and Use Committees of the RIKEN Wako Research Center and Ehime University.

In utero electroporation. Mouse embryos at E14.5 to E15.5 were in-utero electroporated with a pCAG-VSFP Butterfly 1.2 plasmid using a protocol yielding high expression in layer $2 / 3$ pyramidal cells across large part of the cortical hemisphere. Time-pregnant mice (CD-1; ICR, Japan SLC, Inc.) were anesthetized with sodium pentobarbital $(50 \mathrm{mg} / \mathrm{kg}$ body weight, injected intraperitoneally) and a midline laparotomy was performed to expose the uterine horns. Heads of individual embryos were held in a position providing visual access to the dorsal telencephalon and $1-2 \mu \mathrm{L}$ of plasmid $(5 \mu \mathrm{g} / \mu \mathrm{L})$ mixed with the dye Fast Green $(0.05 \%$ w/v; Sigma $)$ was injected into the left lateral ventricle through the uterine wall using a glass capillary. Three electric pulses (43 V, 50 ms; CUY21EDIT, Nepa Gene, Japan) were applied at $950 \mathrm{~ms}$ intervals through forceps-shaped electrodes (CYU650, Nepa Gene) contacting the dorsal (positive electrode) and ventral (negative electrode) telencephalon, with the uterine wall kept moist with PBS $\left(37^{\circ}\right)$ solution. After embryos (sparing the first and last in each horn) were electroporated, the electroporation was repeated a second time using a slightly more anterior site for plasmid injection. Finally, the uterus was moved back to the uterine cavity, incisions of abdominal muscle and skin were closed with sutures and mice were recovered from anesthesia within $1 \mathrm{~h}$ in a small recovery cage on a heat stage.

Slice imaging. Young adult mice (21-30 d) were deeply anesthetized with halothane, and dissected brains quickly transferred into ice-cold artificial cerebral-spinal fluid (ACSF) composed of (in mM): $126 \mathrm{NaCl}, 3 \mathrm{KCl}, 2 \mathrm{CaCl}_{2}, 1 \mathrm{MgSO}_{4}, 1.25 \mathrm{NaH}_{2} \mathrm{PO}_{4}, 24$ $\mathrm{NaHCO}_{3}, 10 \mathrm{D}$-glucose at $\mathrm{pH}$ 7.4. Coronal cortical slices $(300 \mu \mathrm{m})$ were cut using a vibratome (Leica TS1200) and stored in carbogenated ACSF for an initial $30 \mathrm{~min}$ at $32^{\circ} \mathrm{C}$ and later for 0.5 to $4 \mathrm{~h}$ at room temperature $\left(22-25^{\circ} \mathrm{C}\right)$. For experiment, individual slices were transferred into a slice chamber under a 2-photon microscope and superfused with ACSF at $30-32^{\circ} \mathrm{C}$. For extracellular field stimulation a stimulus consisting of five brief current pulses $(0.2 \mathrm{~ms}, 20-70 \mu \mathrm{A})$ at $100 \mathrm{~Hz}$ (Master 8, A.M.P.I) were delivered through a glass microelectrode filled with ACSF and positioned in the granular layer close to the imaged area. The microscope consisted of a 2-photon non-descan microscope featuring fast galvometric mirror scanners (Ultima 4, Prairie) coupled to a TiSaphir laser (Chameleon, Coherent). The microscope was equipped with an auxiliary CCD port and epifluorescence condenser for 1-photon fluorescence imaging. 1-photon imaging employed a feedbackstabilized blue LED (465 nm max; Lex-2, Brain Vision), a cooled CCD camera (QuantumEM, Roper) and optical filters for mCitrine imaging, FF01-483/32-25 (Semrock) for excitation, FF01-542/27-25 for emission (Semrock), FF506-Di03-25 $\times$ 36 (Semrock) for excitation beam splitting. 2-photon imaging was performed in frame or spiral scan at video rate and $64 \times 64$ pixelation using an Olympus $20 \times \mathrm{NA}$ 0.95 water immersion objective. The mCitrine and mKate 2 signals were split and simultaneously recorded using, FF01-542/27-25 for mCitrine emission (Semrock), BLP01-594R-25 for mKate2 emission (Semrock), FF593-Di03-25 × 36 (Semrock) for $\mathrm{mCitrine} / \mathrm{mKate}$ detection splitting.

Animal surgery for in-vivo imaging. Adult mice (30 to 120 day old) were deeply anesthetized with pentobarbital $(0.9 \mathrm{~g} / \mathrm{Kg}$ i.p. $)$ or a combination of $0.3 \mathrm{mg} / \mathrm{kg}$ of medetomidine, $4.0 \mathrm{mg} / \mathrm{kg}$ of midazolam, and $5.0 \mathrm{mg} / \mathrm{kg}$ of butorphanol. Soft tissue was retracted to expose the cranial bone and a metal rod serving as a hold for head fixation during experiments was mounted on the frontal cranial bone with dental cement. The bone over the left somato-sensory cortex was thinned with care, to minimize heat exposure, using a dental ball driller $(2 \mathrm{~mm})$ until the bone became highly transparent (thickness $<30 \mu \mathrm{m}$ ) giving a sharp view of the pial vasculature. Whiskers on the right snout were cut except of the $\mathrm{C} 1$ and D1 whiskers which were trimmed to $15 \mathrm{~mm}$ length. For imaging, the animals were head-fixed in a custommade stereotaxic frame with the body temperature maintained at $37^{\circ} \mathrm{C}$ by means of a feedback-controlled heat pad (Fine Science Tools, Tokyo). For whisker stimulation, a pasteur pipette connected to a fast gated air flow system (Picospritzer III, Parker Hannifin) was adjusted to direct a $100 \mathrm{~ms}$ focal air puff onto one whisker evoking a fast forward (caudal to rostral) deflection. The cortical location of the C1 or D1 barrel was determined by transcranial imaging (see below) in the anesthetized animal. A small craniotomy $(2 \mathrm{~mm}$ ) was performed over the cortical area responsive to the stimulus by preserving the dura mater. The craniotomy was then filled with 0.5 to $1.5 \%$ agarose dissolved in HEPES-buffered ACSF to stabilize the cortical surface and finally covered with a small cover glass.
Optical in-vivo imaging. During experimental sessions animals were kept under deep anaesthesia indicated by absence of foot pinch responses and absence of spontaneous whisking by repeated intraperitonal applications of pentobarbital or a combination anesthetic with $0.3 \mathrm{mg} / \mathrm{kg}$ of medetomidine, $4.0 \mathrm{mg} / \mathrm{kg}$ of midazolam, and $5.0 \mathrm{mg} / \mathrm{kg}$ of butorphanol, respectively, as required. The position of the $\mathrm{C} 1$ or D1 barrel column in somatosensory cortex was determined by dual-channnel, whole hemisphere, transcranial imaging of Butterfly 1.2 using Leica PlanOPO 1.0 and Leica PlanOPO1.6 lenses in tandem and two synchronized CCD cameras (Sensicam, PCO). Excitation light was supplied by a high power halogen lamp (Moritex, Brainvision, Tokyo). The optics included the following optical filters FF01-483/32-25 for mCitrine excitation (Semrock), FF01-542/27-25 for mCitrine emission (Semrock), BLP01594R-25 for mKate2 emission (Semrock), FF506-Di03-25 $\times 36$ (Semrock) as excitation beamsplitter, FF593-Di03-25 × 36 (Semrock) as detection beamsplitter. Image sequences (20 trials) of $4 \mathrm{~s}$ duration and $30 \mathrm{~s}$ interstimulus periode were acquired at $50 \mathrm{fps}(320 \times 240$ pixels $)$ using custom acquisition macros under ImagePro 6.2. Two-photon imaging was performed using either the same setup used for slice recording, but using a NIKON $16 \times$ NA 0.8 water objective, or a setup featuring a fast resonant scanner (NIKON A1R MP+) giving $512 \times 512$ pixel frames at video rate using a NIKON $25 \times$ NA 1.1 water immersion objective and a dispersion-corrected high power Ti-Saphire laser (Mai Tai HP DeepSee, SpectraPhysics) at $950 \mathrm{~nm}$, together with optical filters 535/50 for mCitrine emission (Semrock), 594LP for mKate2 emission (Semrock), 593LP for mCitrine/mKate detection splitting. Imaging trials had a length of $5 \mathrm{~s}$ with the puff stimulus applied after $2 \mathrm{~s}$ or $3 \mathrm{~s}$, repeated $20-32$ times with $30 \mathrm{~s}$ interstimulus period.

Data analysis. Imaging data were analyzed using Matlab 7 image and signal processing toolboxes (Mathworks) and ImagePro6.2 image processing software. 512 $\times 512$ image sequences were down-sampled using a $4 \times 4$ averaging kernel for noise reduction. Absolute mCitrine response images $\left(\Delta \mathrm{F} / \Delta \mathrm{F}_{\max }\right)$ were obtained by subtraction of the average of the pre-stimulus sequence after subtraction of dark offsets and normalization by the maximum absolute response. Fractional response images $\left(\mathrm{F} / \mathrm{F}_{0}\right)$ were obtained by normalizing the sequence by the pre-stimulus average after subtraction of dark offsets. Ratio images $\left(R / R_{0}\right)$ were obtained by dividing the $\mathrm{mKate} 2$ by the mCitrine sequence after offset subtraction and noise gain equalization (see ref. 12) and subsequent normalization by the prestimulus ratio average. Response images were spatially filtered using $8 \times 8$ or $12 \times 12$ Gauss kernels. To visualize the most responsive areas in the image we ranked pixels in the absolute mCitrine response image in the time window of peak response according to $\mathrm{z}$-score and applied a threshold of either 1.5 or 2 . In the case of high magnification, high resolution images we ranked pixels according to absolute brightness in the unfiltered mCitrine fluorescence image and filtered the absolute mCitrine response with a $32 \times 32$ averaging kernel using only pixels that reached a z-score of 2 in absolute brightness. Time traces were extracted from the imaging data using either circular regions of interest or regions of interest comprising pixels surpassing the threshold in absolute response or absolute brightness, respectively, as stated in the text.

1. Ferster, D. \& Spruston, N. Cracking the neuronal code. Science 270, 756-757 (1995).

2. Grillner, S. et al. Intrinsic function of a neuronal network - a vertebrate central pattern generator. Brain Res. Brain Res. Rev. 26, 184-197 (1998).

3. Stanley, G. B. Reading and writing the neural code. Nat. Neurosci. 16, 259-263 (2013).

4. Gray, C. M., Konig, P., Engel, A. K. \& Singer, W. Oscillatory responses in cat visual cortex exhibit inter-columnar synchronization which reflects global stimulus properties. Nature 338, 334-337 (1989).

5. O'Keefe, J. \& Recce, M. L. Phase relationship between hippocampal place units and the EEG theta rhythm. Hippocampus 3, 317-330 (1993).

6. Csicsvari, J., Jamieson, B., Wise, K. D. \& Buzsaki, G. Mechanisms of gamma oscillations in the hippocampus of the behaving rat. Neuron 37, 311-322 (2003).

7. Bao, W. \& Wu, J. Y. Propagating wave and irregular dynamics: spatiotemporal patterns of cholinergic theta oscillations in neocortex in vitro. J. Neurophysiol. 90 , 333-341 (2003).

8. Zochowski, M. R. \& Cohen, L. B. Oscillations in the olfactory bulb carry information about odorant history. J. Neurophysiol. 94, 2667-2675 (2005).

9. Dimitrov, D. et al. Engineering and characterization of an enhanced fluorescent protein voltage sensor. PLoS ONE 2, e440 (2007).

10. Tsutsui, H., Karasawa, S., Okamura, Y. \& Miyawaki, A. Improving membrane voltage measurements using FRET with new fluorescent proteins. Nature Methods 5, 683-685 (2008).

11. Kralj, J. M., Douglass, A. D., Hochbaum, D. R., Maclaurin, D. \& Cohen, A. E. Optical recording of action potentials in mammalian neurons using a microbial rhodopsin. Nat. Methods 9, 90-95 (2012).

12. Akemann, W. et al. Imaging neural circuit dynamics with a voltage-sensitive fluorescent protein. J. Neurophysiol. 108, 2323-2337 (2012).

13. Lam, A. J. et al. Improving FRET dynamic range with bright green and red fluorescent proteins. Nat. Methods 9, 1005-1012 (2012).

14. Perron, A., Mutoh, H., Launey, T. \& Knopfel, T. Red-shifted voltage-sensitive fluorescent proteins. Chem. Biol. 16, 1268-1277 (2009).

15. Knopfel, T., Diez-Garcia, J. \& Akemann, W. Optical probing of neuronal circuit dynamics: genetically encoded versus classical fluorescent sensors. Trends in Neurosciences 29, 160-166 (2006). 
16. Boyden, E. S., Zhang, F., Bamberg, E., Nagel, G. \& Deisseroth, K. Millisecondtimescale, genetically targeted optical control of neural activity. Nat. Neurosci. 8 1263-1268 (2005)

17. Dugue, G. P., Akemann, W. \& Knopfel, T. A comprehensive concept of optogenetics. Prog. Brain Res. 196, 1-28 (2012).

18. Jin, L. et al. Single action potentials and subthreshold electrical events imaged in neurons with a fluorescent protein voltage probe. Neuron 75, 779-785 (2012).

19. Baker, B. J. et al. Genetically encoded fluorescent voltage sensors using the voltagesensing domain of Nematostella and Danio phosphatases exhibit fast kinetics. J. Neurosci. Methods 208, 190-196 (2012).

20. Akemann, W., Mutoh, H., Perron, A., Rossier, J. \& Knopfel, T. Imaging brain electric signals with genetically targeted voltage-sensitive fluorescent proteins. Nat. Methods 7, 643-649 (2010).

21. Lundby, A., Mutoh, H., Dimitrov, D., Akemann, W. \& Knopfel, T. Engineering of a genetically encodable fluorescent voltage sensor exploiting fast Ci-VSP voltagesensing movements. PLoS. ONE. 3, e2514 (2008).

22. Benucci, A., Ringach, D. L. \& Carandini, M. Coding of stimulus sequences by population responses in visual cortex. Nat. Neurosci. 12, 1317-1324 (2009).

23. Slovin, H., Arieli, A., Hildesheim, R. \& Grinvald, A. Long-term voltage-sensitive dye imaging reveals cortical dynamics in behaving monkeys. J. Neurophysiol. $\mathbf{8 8}$, 3421-3438 (2002)

24. Kenet, T., Bibitchkov, D., Tsodyks, M., Grinvald, A. \& Arieli, A. Spontaneously emerging cortical representations of visual attributes. Nature 425, 954-956 (2003).

25. Ferezou, I. et al. Spatiotemporal dynamics of cortical sensorimotor integration in behaving mice. Neuron 56, 907-923 (2007)

26. Petersen, C. C., Grinvald, A. \& Sakmann, B. Spatiotemporal dynamics of sensory responses in layer $2 / 3$ of rat barrel cortex measured in vivo by voltage-sensitive dye imaging combined with whole-cell voltage recordings and neuron reconstructions. J. Neurosci. 23, 1298-1309 (2003).

27. Kubota, M., Miyamoto, A., Hosokawa, Y., Sugimoto, S. \& Horikawa, J. Spatiotemporal dynamics of neural activity related to auditory induction in the core and belt fields of guinea-pig auditory cortex. Neuroreport 23, 474-478 (2012).

28. Farley, B. J. \& Norena, A. J. Spatiotemporal Coordination of Slow-Wave Ongoing Activity across Auditory Cortical Areas. J. Neurosci. 33, 3299-3310 (2013).

29. Friedrich, R. W., Habermann, C. J. \& Laurent, G. Multiplexing using synchrony in the zebrafish olfactory bulb. Nat. Neurosci. 7, 862-871 (2004)

30. Denk, W., Strickler, J. H. \& Webb, W. W. Two-photon laser scanning fluorescence microscopy. Science 248, 73-76 (1990).

31. Kuhn, B., Denk, W. \& Bruno, R. M. In vivo two-photon voltage-sensitive dye imaging reveals top-down control of cortical layers 1 and 2 during wakefulness. Proc. Natl. Acad. Sci. U. S. A 105, 7588-7593 (2008).

32. Fisher, J. A. et al. Two-photon excitation of potentiometric probes enables optical recording of action potentials from mammalian nerve terminals in situ. J. Neurophysiol. 99, 1545-1553 (2008).

33. Acker, C. D., Yan, P. \& Loew, L. M. Single-voxel recording of voltage transients in dendritic spines. Biophys. J. 101, L11-L13 (2011)

34. Yan, P. et al. Palette of fluorinated voltage-sensitive hemicyanine dyes. Proc. Natl. Acad. Sci. U. S. A 109, 20443-20448 (2012)

35. Ahrens, K. F., Heider, B., Lee, H., Isacoff, E. Y. \& Siegel, R. M. Two-photon scanning microscopy of in vivo sensory responses of cortical neurons genetically encoded with a fluorescent voltage sensor in rat. Front Neural Circuits. 6, 15 (2012).

36. Drobizhev, M., Makarov, N. S., Tillo, S. E., Hughes, T. E. \& Rebane, A. Twophoton absorption properties of fluorescent proteins. Nat. Methods 8, 393-399 (2011)
37. Zhu, J. J. \& Connors, B. W. Intrinsic firing patterns and whisker-evoked synaptic responses of neurons in the rat barrel cortex. J. Neurophysiol. 81, 1171-1183 (1999).

38. Brecht, M., Roth, A. \& Sakmann, B. Dynamic receptive fields of reconstructed pyramidal cells in layers 2 and 3 of rat somatosensory barrel cortex. J. Physiol 553, 243-265 (2003).

39. Smetters, D., Majewska, A. \& Yuste, R. Detecting action potentials in neuronal populations with calcium imaging. Methods 18, 215-221 (1999).

40. Cossart, R., Ikegaya, Y. \& Yuste, R. Calcium imaging of cortical networks dynamics. Cell Calcium 37, 451-457 (2005)

41. Sato, T. R., Gray, N. W., Mainen, Z. F. \& Svoboda, K. The Functional microarchitecture of the mouse barrel cortex. PLoS Biology 5, 1440-1452 (2007).

42. Berger, T. et al. Combined voltage and calcium epifluorescence imaging in vitro and in vivo reveals subthreshold and suprathreshold dynamics of mouse barrel cortex. J. Neurophysiol. 97, 3751-3762 (2007).

43. Akemann, W., Lundby, A., Mutoh, H. \& Knopfel, T. Effect of Voltage Sensitive Fluorescent Proteins on Neuronal Excitability. Biophysical Journal 96, 3959-3976 (2009).

44. Helmchen, F. \& Denk, W. Deep tissue two-photon microscopy. Nat. Methods 2, 932-940 (2005).

45. Baker, B. J. et al. Three fluorescent protein voltage sensors exhibit low plasma membrane expression in mammalian cells. Journal of Neuroscience Methods 161, 32-38 (2007).

46. Papagiakoumou, E. et al. Scanless two-photon excitation of channelrhodopsin-2. Nat. Methods 7, 848-854 (2010).

47. Gobel, W., Kampa, B. M. \& Helmchen, F. Imaging cellular network dynamics in three dimensions using fast 3D laser scanning. Nat. Methods 4, 73-79 (2007).

48. Katona, G. et al. Fast two-photon in vivo imaging with three-dimensional random-access scanning in large tissue volumes. Nat. Methods 9, 201-208 (2012).

\section{Acknowledgements}

We thank Nisha Jose for assistance with in-utero electroporation of VSFP Butterfly 1.2 plasmid and Miyoko Seike for supporting analysis.

\section{Author contributions}

T.K., M.S., W.A., T.I. designed research; W.A., M.S., H.M., N.H. performed experiments W.A., M.S., H.M., N.H., T.K. analysed data; W.A., M.S., H.M., T.I., N.H., T.K. discussed results; W.A., T.K., M.S. wrote the manuscript with help from all authors.

\section{Additional information}

Competing financial interests: The authors declare no competing financial interests.

How to cite this article: Akemann, W. et al. Two-photon voltage imaging using a genetically encoded voltage indicator. Sci. Rep. 3, 2231; DOI:10.1038/srep02231 (2013).

cc) (i) $\Theta$ This work is licensed under a Creative Commons Attribution-

NonCommercial-NoDerivs 3.0 Unported license. To view a copy of this license, visit http://creativecommons.org/licenses/by-nc-nd/3.0 